\title{
Interobserver agreement of skeletal muscle mass measurement on head and neck CT imaging at the level of the third cervical vertebra
}

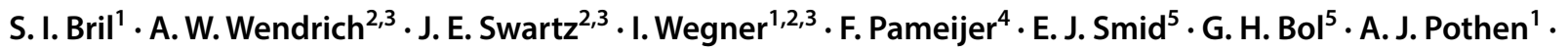 \\ R. de Bree ${ }^{1}$ (I)
}

Received: 25 December 2018 / Accepted: 18 January 2019 / Published online: 28 January 2019

(c) The Author(s) 2019

\begin{abstract}
Objectives Skeletal muscle mass (SMM) is most often assessed in cancer patients on abdominal computed tomography (CT) imaging at the level of the third lumbar vertebra (L3). Abdominal CT imaging is not routinely performed in head and neck cancer (HNC) patients. Recently, a novel method to assess SMM on a single transversal CT slice at the level of the third cervical vertebra (C3) was published. The objective of this study was to assess the robustness of this novel C3 measurement method in terms of interobserver agreement.

Patients and methods Patients diagnosed with locally advanced head and neck squamous cell carcinoma (LA-HNSCC) at our center between 2007 and 2011 were evaluated. Fifty-four patients with were randomly selected for analysis. Six observers independently measured the cross-sectional muscle area (CSMA) at the level of C 3 using a predefined, written protocol as instruction. Interobserver agreement was assessed using intraclass correlation coefficients (ICCs), a Bland-Altman plot and Fleiss' kappa (к).

Results The agreement in vertebra selection between all observers was excellent (Fleiss' $\kappa: 0.96$ ). There was a substantial agreement between all observers in single slice selection (Fleiss' $\kappa: 0.61$ ). For all CSMA measurements, ICCs were excellent $(0.763-0.969$; all $p<0.001)$. The Bland-Altman plot showed good agreement between measurements, with narrow limits of agreement.

Conclusion Interobserver agreement for SMM measurement at the level of C3 was excellent. Assessment of SMM at the level of $\mathrm{C} 3$ is easy and robust and can performed on routinely available imaging in HNC patients.
\end{abstract}

Keywords Head and neck neoplasms $\cdot$ Sarcopenia $\cdot$ Body composition $\cdot$ Computed tomography $\cdot$ Observer variation

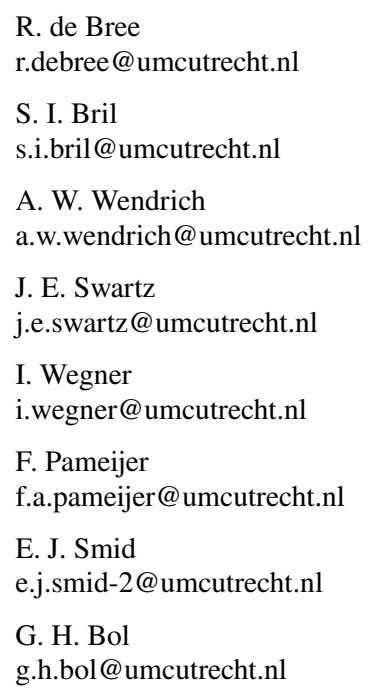

1 Department of Head and Neck Surgical Oncology, University Medical Center Utrecht, House Postal Number Q.04.5.300, Heidelberglaan 100, PO BOX 85500, 3584 CX Utrecht, The Netherlands

2 Department of Otorhinolaryngology - Head and Neck Surgery, University Medical Center Utrecht, Heidelberglaan 100, 3584 CX Utrecht, The Netherlands

3 Brain Center Rudolf Magnus, University Medical Center Utrecht, Heidelberglaan 100, 3584 CX Utrecht, The Netherlands

4 Department of Radiology, University Medical Center Utrecht, Heidelberglaan 100, 3584 CX Utrecht, The Netherlands

5 Department of Radiation Oncology, University Medical Center Utrecht, Heidelberglaan 100, 3584 CX Utrecht, The Netherlands 


\begin{tabular}{ll}
\multicolumn{2}{l}{ Abbreviations } \\
SMM & Skeletal muscle mass \\
HNC & Head and neck cancer \\
C3 & Third cervical vertebra \\
L3 & Third lumbar vertebra \\
CSMA & Cross-sectional muscle area \\
HU & Hounsfield unit \\
Lumbar SMI & Lumbar skeletal muscle index
\end{tabular}

\section{Introduction}

Body composition increasingly is a subject of interest in medical research. An abnormal body composition, such as a decreased lean body mass and/or increased adipose tissue mass, may have a profound influence on treatment outcome and (disease free) survival of patients with a variety of illnesses [1-3]. Skeletal muscle mass (SMM) is the largest component of lean body mass [4]. In cancer patients, a low SMM, sometimes referred to as sarcopenia, has specifically been associated with a higher incidence of chemotherapyrelated toxicity, postoperative complications, longer hospital stay, increased healthcare-related expenditures, and lower disease free and overall survival [5-10]. This relationship has been shown in breast, colorectal, hepato-pancreaticobiliary, renal, and lung cancer, amongst others [11-15].

In cancer patients, SMM is most often assessed on abdominal computed tomography (CT) imaging at the level of the third lumbar vertebra (L3) [16]. This method is based on research using whole-body magnetic resonance imaging (MRI), in which has been shown that cross-sectional skeletal muscle area (CSMA) on a single transversal slice at the level of L3 is strongly correlated with total skeletal muscle volume as measured using whole-body MRI [17, 18]. The CSMA at the level of L3 is commonly normalized for stature, which results in the lumbar skeletal muscle index (lumbar SMI) [11]. This value is used as an indication of total SMM.

Abdominal CT imaging is often routinely performed in most types of cancer during diagnostic work-up and followup. In these patients, SMM measurement can be performed on abdominal CT imaging without the need for additional imaging or other diagnostics. However, abdominal CT imaging is not routinely performed in head and neck cancer (HNC) patients [19]. Recently, a novel method to assess SMM on a single transversal CT slice at the level of the third cervical vertebra (C3) was published [20]. Using this method, skeletal muscle mass is assessed measuring the CSMA of the paravertebral muscles and the sternocleidomastoid muscles at the level of the $\mathrm{C} 3$ vertebra. This method allows for evaluation of SMM in HNC patients on routinely performed imaging, in a similar manner as is used in patients with other types of cancer. This measurement method for
SMM was recently used in three studies in head and neck cancer patients [21-23].

To be clinically useful, the $\mathrm{C} 3$ measurement method of SMM has to provide similar results when used by different observers. The aim of this study was to evaluate the interobserver agreement of SMM measurement at the level of C3. The robustness of the $\mathrm{C} 3$ measurement method was investigated in terms of the vertebra selection, the exact single slice selection, and the correspondence in CSMA measurements between all observers.

\section{Patients and methods}

\section{Ethical approval}

The design of this study was approved by the Medical Ethical Research Committee of our center (approval ID 14-544/ C). All procedures in this study were in accordance with the ethical standards of the institutional and/or national research committee and with the 1964 Helsinki declaration and its later amendments or comparable ethical standards. All data were retrieved retrospectively. Measurements of SMM were performed on coded CT scans.

\section{Patients and study design}

Patients diagnosed with locally advanced head and neck squamous cell carcinoma (LA-HNSCC) at the our center between 2007 and 2011 were evaluated for this study. Fiftyfour patients with pre-treatment head and neck CT imaging were randomly selected for inclusion. Other parameters, including length and weight at the time of imaging, sex, age, tumor localization, and clinical TNM stage (7th edition), were retrospectively retrieved [24].

\section{Imaging protocol and analysis}

All patients underwent contrast-enhanced CT scanning prior to radiation treatment on a Philips Brilliance iCT scanner (Philips Healthcare, Best, The Netherlands) using a standardized protocol for HNC patients. The imaging was performed in treatment position in a radiotherapy immobilization mask. Scanning parameters included slice thickness $1 \mathrm{~mm}$ with a $2 \mathrm{~mm}$ interslice gap.

\section{Measurement of SMM}

Delineation of CSMA was manually performed using the Volumetool v.1.6.5 Research Software Package, designed in our center as an image evaluation, registration, and delineation system for radiotherapy planning [25]. Hounsfield Unit (HU) thresholds for skeletal muscle tissue were 
-29 and $+150 \mathrm{HU}$; the area within these HU thresholds was defined as the CSMA. Delineation of CSMA was performed independently by six observers: one experienced head and neck radiologist, one experienced head and neck radiation oncologist, and four medically trained researchers from the departments of Head and Neck Surgical Oncology, and Otorhinolaryngology Head and Neck Surgery. A predefined, written protocol for single slice selection and CSMA was provided, as described in the recently published study by Swartz et al. [20]. In brief, the first slice when scrolling from caudal to cranial direction to show both transverse processes and the entire vertebral arc of the third cervical vertebra had to be selected. Contours of the paravertebral muscles (PVM) and both sternocleidomastoid (SCM) muscles were manually traced. This study was performed without a training data set, as to simulate the use of this measurement method as if it were adopted from an external research paper.

All observers independently selected the slice they deemed to be correct from the CT scan, and delineated CSMA. Observers were blinded to their own and each other's results. After delineation of CSMA at C3 was finished by all observers, CSMA was automatically retrieved from Volumetool. Vertebra and single slice selection were investigated by reopening all scans after CSMA retrieval and analysis was finished; the selected vertebra and single slice location were noted. Total CSMA at C3 was calculated as the sum of the CSMA of the PVM and both SCM muscles. Figure 1 shows an example of CSMA delineation at the level of $\mathrm{C} 3$.

All statistical analyses were performed using the IBM SPSS Statistics version 21.0 software package (Chicago, Illinois, USA). A test for normality (Shapiro-Wilk test) was used to assess whether continuous variables were normally distributed. Continuous data are represented as mean \pm standard deviation (SD). Categorical data are represented as a number and percentage of total. Differences in skeletal muscle area measurements between observers were calculated using a repeated measures ANOVA. Agreement between different observers was assessed by calculating Fleiss' kappa (к) and intraclass correlation coefficients (ICCs) using a two-way mixed single measures model with absolute agreement and. The $\kappa$ values were graded as slight $(0.01-0.20)$, fair $(0.21-0.40)$, moderate (0.41-0.60), substantial (0.61-0.80), or almost perfect (0.81-0.99) agreement [26]. The ICCs were rated as poor (0.00-0.49), fair to good (0.50-0.74), and excellent (0.75-1.00) [27]. Bland-Altman plots were constructed to visualize agreement [28]. Results were considered statistically significant if the $p$ value was less than 0.05 .

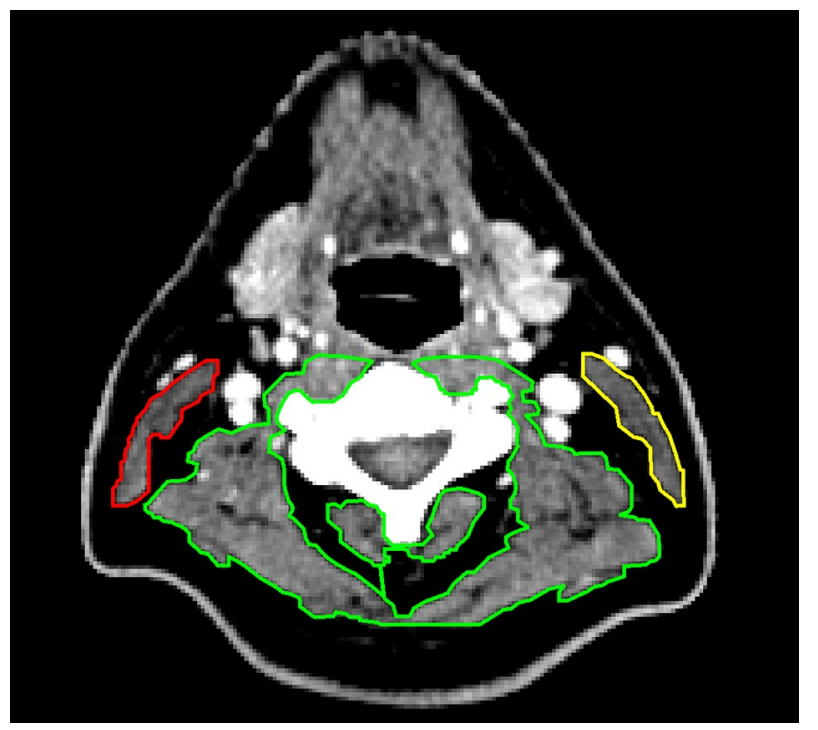

Fig. 1 Example of delineation of skeletal muscle at the level of C3. The paravertebral muscles are delineation in green. The left sternocleidomastoid muscle is delineated in yellow, and the right sternocleidomastoid muscle in red

\section{Results}

\section{Patient characteristics}

Baseline characteristics of the 54 included patients are shown in Table 1. Patients were predominantly male and mostly presented with lymph node positive, stage IV disease.

\section{Vertebra and single slice selection}

Table 2 shows the results of the vertebra and single slice selection analysis. For the vertebra selection, the overall correspondence between all observers was near perfect (Fleiss' $\kappa: 0.96, p<0.001)$. The correct vertebra $(\mathrm{C} 3)$ was identified in almost all patients by all observers; in five patients, one observers chose a different vertebra, and in one patient, two observers chose a different vertebra. Either $\mathrm{C} 2$ or $\mathrm{C} 4$ was selected in these patients.

There was more variation in exact single slice selection. The overall correspondence between all observers was substantial (Fleiss' $\kappa: 0.61, p<0.001$ ). In $22.2 \%$ of patients, all observers chose the same identical slice to delineate, while in the other $77.8 \%$, at least one observer chose a different slice. In $79.3 \%$ of the cases, where a different slice from the majority was chosen, the different slices were located directly above or below the slice that the majority of observers chose. 
Table 1 Baseline patient characteristics

\begin{tabular}{lc}
\hline & Total $n=54$ \\
\hline Sex & \\
Male & $36(66.7 \%)$ \\
Female & $18(33.3 \%)$ \\
Age & $56.8(7.3)$ \\
Weight & $67.8(13.2)$ \\
BMI & $22.9(3.8)$ \\
T-stage & \\
T1-2 & $13(24.1 \%)$ \\
T3-4 & $41(75.9 \%)$ \\
N-stage & \\
N0 & $5(9.3 \%)$ \\
N+ & $49(90.7 \%)$ \\
Clinical TNM stage & \\
Stage III & $5(9.3 \%)$ \\
Stage IV & $49(90.7 \%)$ \\
Tumor site & \\
Oral cavity & $6(11.1 \%)$ \\
Nasopharyngeal & $10(18.5 \%)$ \\
Oropharyngeal & $20(37.0 \%)$ \\
Hypopharyngeal & $8(14.8 \%)$ \\
Laryngeal & $5(9.3 \%)$ \\
Other & $5(9.3 \%)$ \\
\hline
\end{tabular}

Continuous data are represented as mean (SD). Categorical data are represented as number (\% of total)

${ }^{\text {a }}$ Staged according to the AJCC Cancer Staging Manual 7th edition

${ }^{\mathrm{b}}$ Other $=$ sinus $(n=1)$, proximal esophagus $(n=2)$, and multiple $(n=2)$

Table 2 Vertebra and single CT slice selection

\begin{tabular}{lll}
\hline & Vertebra $(n=54)$ & Slice $(n=54)$ \\
\hline All observers same & $49(88.9 \%)$ & $12(22.2 \%)$ \\
One observer different & $5(9.1 \%)$ & $16(29.6 \%)$ \\
Two observers different & $1(1.9 \%)$ & $12(22.2 \%)$ \\
Three observers different & - & $14(25.9 \%)$ \\
\hline
\end{tabular}

Values shown as number (\% of total)

\section{Cross-sectional skeletal muscle area measurements}

Mean CSMA of the PVM, left SCM, and right SCM and total CSMA at $\mathrm{C} 3$ are shown in Table 3. Most CSMA measurements were normally distributed (Shapiro-Wilk: $p>0.05)$. There was a significant difference between the observers in CSMA measurements (repeated measures ANOVA: $p<0.001$ ). Actual differences between CSMA measurements of all observers were small; the largest mean difference between observers was $1.48 \mathrm{~cm}^{2}$ (observer 6-observer 4). For all CSMA measurements, ICCs were excellent $(0.763-0.969$; all $p<0.001)$, showing good conformity between measurements of different observers, as visualized in Fig. 2. Figure 3 shows a combined Bland-Altman plot of total CSMA at C3 measurements of the individual observers and their difference to the overall mean CSMA at C3 measurements. The mean of the standard deviation of the difference between observer CSMA measurements and mean CSMA measurement was used to calculate the limits of agreement ( $95 \%$ confidence interval). There appears to be an element of systemic bias in CSMA measurement at C3, with some observers having more than $5 \%$ measurements outside of the $95 \%$ limits of agreement, and some observers systemically deviate one way (higher or lower) from the mean CSMA measurement. Actual differences are small.

\section{Discussion}

This study aimed to examine the robustness of the C3 measurement method for SMM in terms of interobserver agreement and specific vertebra and single slice selection by multiple observers. This study was performed without a training data set and limited formal training, to simulate the use of the $\mathrm{C} 3$ measurement method for SMM as if it were adopted from an external research paper.

The interobserver agreement for all different CSMA measurements was excellent. The measurement of CSMA of PVM appears to be most uniform. There is some difference between observers in measurement of the CSMA of the SCM muscles; however, actual differences remain small. The previous research has shown that total CSMA at C3 was significantly better correlated with CSMA at L3 than the CSMA of the PVM only with CSMA at L3 [20].

The level of $\mathrm{C} 3$ is easily identifiable for both researchers and clinicians, with only incidental selection of a different level. There was more variation in the actual single slice selection, perhaps due to the lack of a training data set and little informal training received prior to delineation of scans. This may also be due to the thin slices $(1 \mathrm{~mm})$ and small (2 mm) interslice gaps of the CT imaging used in this study. In most cases, where an observer had selected a different slice than the majority of the observers, the different slices were indeed directly above or below of the slice the majority chose, which corresponds with a $3 \mathrm{~mm}$ difference in location. Probably, in these cases, the accidental selection of a different slice should not greatly influence CSMA measurements. However, it may still be advisable to include a training data set when starting to use the $\mathrm{C} 3$ measurement method to allow for a learning period in delineation of skeletal muscle and single slice selection.

In recent years, SMM has widely been researched in cancer patients, using a measurement of skeletal muscle area at the level of L3 on CT imaging as an indicator of total SMM 
Table 3 Cross-sectional muscle area (CSA) at the level of C3

\begin{tabular}{|c|c|c|c|c|c|c|c|c|c|}
\hline & Total $(n=324)$ & $\begin{array}{l}\text { Obs. } 1 \\
(n=54)\end{array}$ & $\begin{array}{l}\text { Obs. } 2 \\
(n=54)\end{array}$ & $\begin{array}{l}\text { Obs. } 3 \\
(n=54)\end{array}$ & $\begin{array}{l}\text { Obs. } 4 \\
(n=54)\end{array}$ & $\begin{array}{l}\text { Obs. } 5 \\
(n=54)\end{array}$ & $\begin{array}{l}\text { Obs. } 6 \\
(n=54)\end{array}$ & $p$ value* & $\begin{array}{l}\text { ICC } \\
(95 \% \mathrm{CI})\end{array}$ \\
\hline CSMA PVM & $28.03(6.63)$ & $28.52(6.84)$ & $27.95(6.52)$ & $27.45(6.83)$ & $27.53(6.56)$ & $28.15(6.67)$ & $28.59(6.59)$ & $<0.001$ & $\begin{array}{l}0.969(0.953- \\
0.981)\end{array}$ \\
\hline $\begin{array}{l}\text { CSMA left } \\
\text { SCM }\end{array}$ & $2.62(1.30)$ & $2.71(1.35)$ & $2.56(1.40)$ & $2.61(1.25)$ & $2.47(1.22)$ & $2.68(1.30)$ & $2.70(1.32)$ & $<0.001$ & $\begin{array}{l}0.821(0.754 \\
0.880)\end{array}$ \\
\hline $\begin{array}{l}\text { CSMA right } \\
\text { SCM }\end{array}$ & $2.44(1.34)$ & $2.43(1.46)$ & $2.22(1.62)$ & $2.58(1.18)$ & $2.33(1.24)$ & $2.54(1.23)$ & $2.53(1.32)$ & $<0.001$ & $\begin{array}{l}0.763(0.680- \\
0.837)\end{array}$ \\
\hline $\begin{array}{l}\text { Total CSMA } \\
\text { at C3 }\end{array}$ & 33.09 (8.07) & $33.66(8.28)$ & $32.73(8.13)$ & $32.64(8.40)$ & $32.34(7.80)$ & $33.37(8.22)$ & $33.82(7.82)$ & $<0.001$ & $\begin{array}{l}0.969(0.952- \\
0.980)\end{array}$ \\
\hline
\end{tabular}

All values are represented as mean (SD)

CSA cross-sectional muscle area, $P V M$ paravertebral muscles, SCM sternocleidomastoid muscle, Obs observer

${ }^{*} p$ value calculated using a repeated measures ANOVA with a Greenhouse-Geisser correction for sphericity

Fig. 2 Intra-class correlation plot for measurement of total CSMA measurement at $\mathrm{C} 3$.

The correspondence between measurements is visualized in the intra-class correlation plot

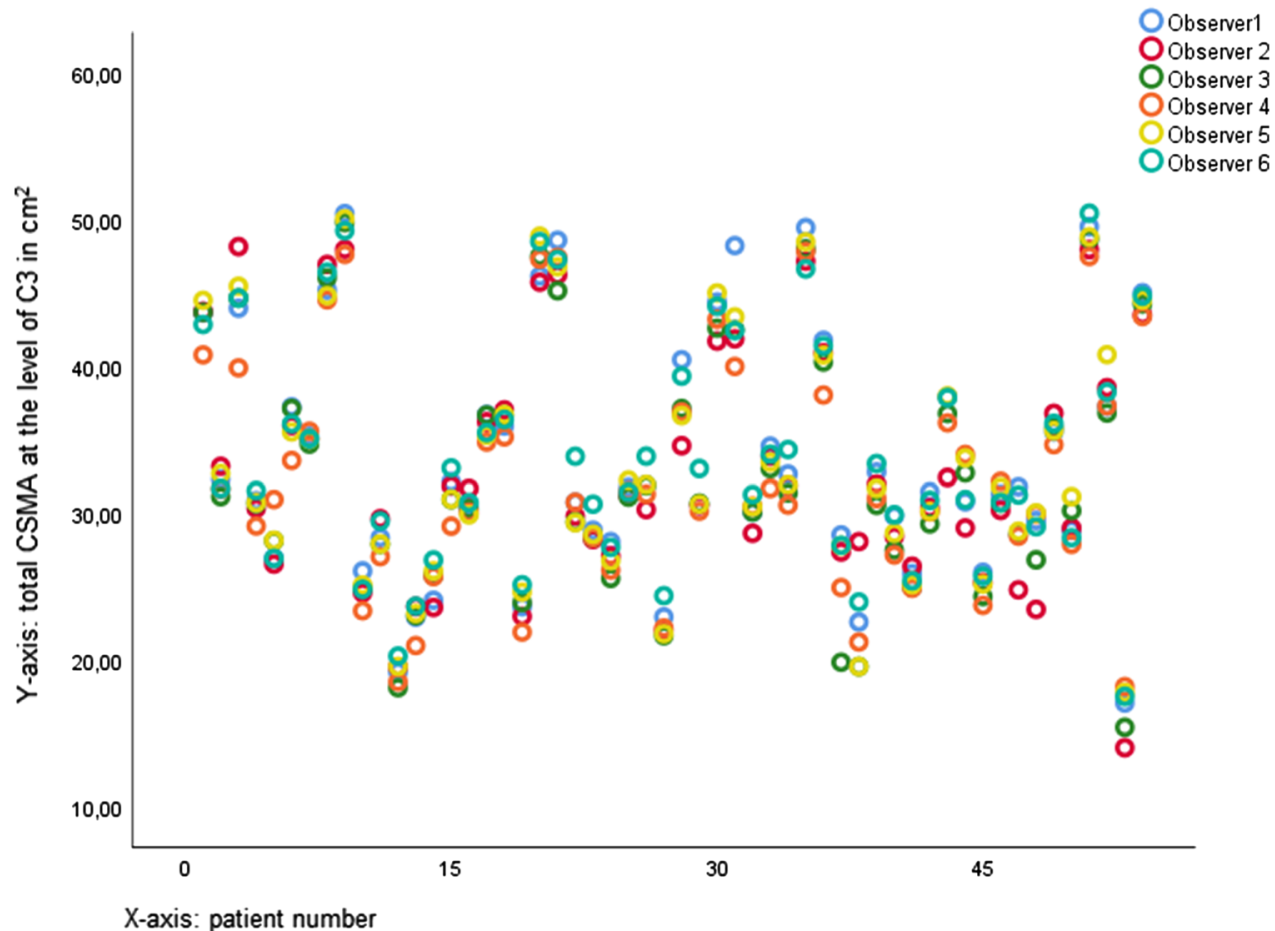

[11]. Imaging at the level of L3 is not routinely performed in HNC patients, so SMM measurement at the level of L3 is not always clinically applicable in HNC [19]. The SMM measurement method at the level of $\mathrm{C} 3$ provides a reliable and robust alternative to SMM measurement at the level of L3, allowing for broad research into the predictive and prognostic effect of sarcopenia in HNC.

Patients with HNC often present with signs of malnutrition and as such are at risk of developing sarcopenia [29]. Adverse outcomes associated with sarcopenia, such as chemotherapy-related toxicity and wound healing problems, are highly prevalent in HNC [30]. It can be anticipated that both are at least partly related to sarcopenia.
The first study using the $\mathrm{C} 3$ measurement method to assess SMM in HNC patients undergoing chemoradiotherapy found that low SMM was an independent predictor of the occurrence of chemotherapy dose-limiting toxicity [21]. A recent study in $\mathrm{HNC}$ patients undergoing laryngectomy for laryngeal cancer showed that low SMM, as measured at the level of L3, was an independent predictor of the occurrence of a pharyngocutaneous fistula and of the occurrence of any wound complication [31]. In this study, 122 patients who had undergone a total laryngectomy were retrospectively evaluated for inclusion; 70 (57\%) had abdominal imaging available for analysis. It is likely that all or almost all HNC patients will undergo CT or MRI 
Fig. 3 Bland-Altman plot for agreement of total CSMA measurement at $\mathrm{C} 3$ between all observers. Bland-Altman plots showing agreement between measurements of observers. The solid lines depict the mean and 95\% limits of agreement in the Bland-Altman plot

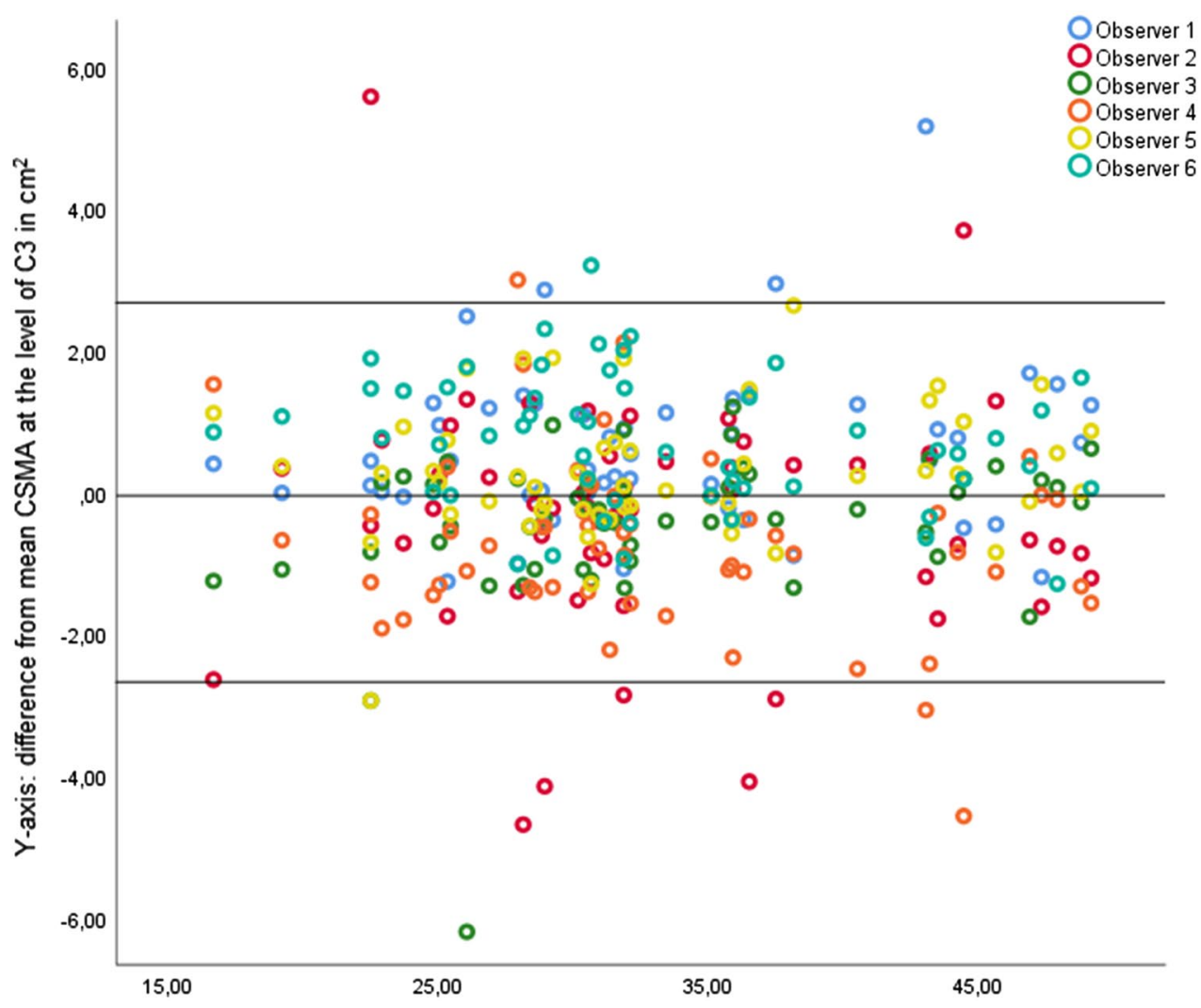

$\mathrm{X}$-axis: mean total CSMA at the level of $\mathrm{C} 3$ in $\mathrm{cm}^{2}$ imaging of the head and neck area during the diagnostic process and follow-up [19]. The C3 measurement method allows the investigation of body composition in nearly all HNC patients without the need for extra diagnostics. A recent study in patients undergoing laryngectomy, where SMM was measured at the level of $\mathrm{C} 3$, showed that low SMM was a predictor of the occurrence of a pharyngocutaneous fistula [23]. Preoperative low SMM also was a strong negative prognostic factor for overall survival after laryngectomy [23]. In this retrospective study, 235 out of 245 patients could be included for analysis due to measurement of SMM at the level of C3.

Future research is still needed to clarify whether the adverse effects of low SMM are prognostic only, or if these adverse effects can be overturned. In the future, HNC patients at high risk of adverse outcomes related to low SMM or sarcopenia might benefit from additional supportive treatment or individualized primary treatment. Several possible interventions may be considered, such as altered chemotherapy dosing [32], prehabilitation before surgery [33], enhanced recovery after surgery [34], intensive physiotherapy [35], and additional nutritional support [36]. A measurement of SMM at the level of C3 might be used as a screening tool for low SMM in HNC cancer patients at diagnosis.

\section{Limitations}

There are some limitations to this study that need to be addressed. No training data set or formal training was given to the observers in this study. Although this study setup was chosen, because it may best mimic the clinical adaptation and use of the $\mathrm{C} 3$ measurement method by other institutions, it may also explain some of the differences in the measurements and in the vertebra and single slice selections. For further use in research and clinical work, a training data set for researchers learning skeletal muscle area measurement may limit these differences. Second, only CT imaging was used in this study to assess SMM, as is usually done in other studies in cancer patients that assess SMM at the level of L3. Some HNC patients will only undergo MRI imaging of the head and neck area during the diagnostic process. It should still be evaluated whether CT and MRI can be used interchangeably for SMM assessment or that some form of modification of the method is necessary. Finally, most variation in CSA measurements was seen in the SCM muscles. This can at least partially be explained by lymph node metastasis close to or invading in the SCM muscles. Most patients included in this study had advanced, lymph node positive disease, 
which may make accurate delineation of the muscle more difficult. In daily clinical practice, approximately twothirds of HNC patients present with advanced stage disease (large tumor and/or lymph node positive) [37]. Thus, this study population provides an accurate reflection of the clinical use of the $\mathrm{C} 3$ measurement method. Because previous research showed that the addition of the CSA of the SCM muscles was beneficial for a prediction model, and because actual differences were small and ICCs could still be classified as excellent, it is justified to include the CSA of the SCM muscles in the total CSA at C3 in patients with lymph node positive disease.

\section{Conclusion}

Interobserver agreement is excellent for SMM measurement on head and neck CT imaging at the level of C3, as tested in a setting that mimics a clinical setting. The $\mathrm{C} 3$ measurement method for SMM is robust, easy to use and can be done on routinely performed CT imaging of the head and neck area. It allows for retrospective and prospective research into the predictive and prognostic value of low SMM in the vast majority of HNC patients, as well as use in possible future trials.

Funding This research received a grant from the Michel Keijzer Fonds, a not for profit fund managed by the Dutch Head and Neck Cancer patient support group $(\mathrm{PVHH})$.

\section{Compliance with ethical standards}

Conflict of interest The authors declare that they have no conflict of interest.

Ethical approval All procedures in this study were in accordance with the ethical standards of the institutional and/or national research committee and with the 1964 Helsinki declaration and its later amendments or comparable ethical standards. The design of this study was approved by the Medical Ethical Research Committee of our center (approval ID $14-544 / C)$.

Informed consent A formal informed consent procedure was waived due to the retrospective nature of this study.

OpenAccess This article is distributed under the terms of the Creative Commons Attribution 4.0 International License (http://creativeco mmons.org/licenses/by/4.0/), which permits unrestricted use, distribution, and reproduction in any medium, provided you give appropriate credit to the original author(s) and the source, provide a link to the Creative Commons license, and indicate if changes were made.

\section{References}

1. Cruz-Jentoft AJ, Baeyens JP, Bauer JM, Boirie Y, Cederholm T, Landi F et al (2010) Sarcopenia: European consensus on definition and diagnosis: report of the European Working Group on Sarcopenia in Older People. Age Ageing 39(4):412-423. https:// doi.org/10.1093/ageing/afq034

2. Wannamethee SG, Atkins JL (2015) Muscle loss and obesity: the health implications of sarcopenia and sarcopenic obesity. Proc Nutr Soc 74(4):405-412. https://doi.org/10.1017/S002966511 500169X

3. Janssen I, Heymsfield SB, Ross R (2002) Low relative skeletal muscle mass (sarcopenia) in older persons is associated with functional impairment and physical disability. J Am Geriatr Soc 50(5):889-896

4. Prado CM, Heymsfield SB (2014) Lean tissue imaging: a new era for nutritional assessment and intervention. J Parenter Enter Nutr 38(8):940-953. https://doi.org/10.1177/0148607114550189

5. Rier HN, Jager A, Sleijfer S, Maier AB, Levin MD (2016) The prevalence and prognostic value of low muscle mass in cancer patients: a review of the literature. Oncologist 21(11):1396-1409. https://doi.org/10.1634/theoncologist.2016-0066

6. Shachar SS, Williams GR, Muss HB, Nishijima TF (2016) Prognostic value of sarcopenia in adults with solid tumours: a metaanalysis and systematic review. Eur J Cancer 57:58-67. https:// doi.org/10.1016/j.ejca.2015.12.030

7. Levolger S, van Vugt JL, de Bruin RW, IJzermans JN (2015) Systematic review of sarcopenia in patients operated on for gastrointestinal and hepatopancreatobiliary malignancies. Br J Surg 102(12):1448-1458. https://doi.org/10.1002/bjs.9893

8. Jones K, Gordon-Weeks A, Coleman C, Silva M (2017) Radiologically determined sarcopenia predicts morbidity and mortality following abdominal surgery: a systematic review and metaanalysis. World J Surg 41(9):2266-2279. https://doi.org/10.1007/ s00268-017-3999-2

9. Lieffers JR, Bathe OF, Fassbender K, Winget M, Baracos VE (2012) Sarcopenia is associated with postoperative infection and delayed recovery from colorectal cancer resection surgery. Br J Cancer 107(6):931-936. https://doi.org/10.1038/bjc.2012.350

10. Van Vugt JLA, Buettner S, Levolger S, Coebergh van der Braak RRJ, Suker M, Gaspersz MP et al (2017) Low skeletal muscle mass is associated with increased hospital expenditure in patients undergoing cancer surgery of the alimentary tract. PLoS One 12(10):e0186547. https://doi.org/10.1371/journal.pone.0186547

11. Prado CM, Lieffers JR, McCargar LJ, Reiman T, Sawyer MB, Martin L et al (2008) Prevalence and clinical implications of sarcopenic obesity in patients with solid tumours of the respiratory and gastrointestinal tracts: a population-based study. Lancet Oncol 9(7):629-635. https://doi.org/10.1016/S1470-2045(08)70153-0

12. Mourtzakis M, Prado CM, Lieffers JR, Reiman T, McCargar LJ, Baracos VE (2008) A practical and precise approach to quantification of body composition in cancer patients using computed tomography images acquired during routine care. Appl Physiol Nutr Metab 33(5):997-1006. https://doi.org/10.1139/H08-075

13. Sjoblom B, Gronberg BH, Benth JS, Baracos VE, Flotten O, Hjermstad MJ et al (2015) Low muscle mass is associated with chemotherapy-induced haematological toxicity in advanced nonsmall cell lung cancer. Lung Cancer 90(1):85-91. https://doi. org/10.1016/j.lungcan.2015.07.001

14. Cushen SJ, Power DG, Teo MY, MacEneaney P, Maher MM, McDermott R et al (2017) Body composition by computed tomography as a predictor of toxicity in patients with renal cell carcinoma treated with sunitinib. Am J Clin Oncol 40(1):47-52. https ://doi.org/10.1097/COC.0000000000000061 
15. Levolger S, van Vledder MG, Muslem R, Koek M, Niessen WJ, de Man RA et al (2015) Sarcopenia impairs survival in patients with potentially curable hepatocellular carcinoma. J Surg Oncol 112(2):208-213

16. Carneiro IP, Mazurak VC, Prado CM (2016) Clinical implications of sarcopenic obesity in cancer. Curr Oncol Rep 18(10):62. https ://doi.org/10.1007/s11912-016-0546-5

17. Shen W, Punyanitya M, Wang Z, Gallagher D, St-Onge MP, Albu $\mathrm{J}$ et al (2004) Total body skeletal muscle and adipose tissue volumes: estimation from a single abdominal cross-sectional image. J Appl Physiol 97(6):2333-2338

18. Schweitzer L, Geisler C, Pourhassan M, Braun W, Gluer CC, Bosy-Westphal A et al (2015) What is the best reference site for a single MRI slice to assess whole-body skeletal muscle and adipose tissue volumes in healthy adults? Am J Clin Nutr 102(1):58 65. https://doi.org/10.3945/ajcn.115.111203

19. Gregoire V, Lefebvre JL, Licitra L, Felip E, Group E-E-EGW (2010) Squamous cell carcinoma of the head and neck: EHNSESMO-ESTRO clinical practice guidelines for diagnosis, treatment and follow-up. Ann Oncol 21 (Suppl 5):v184-6. https://doi. org/10.1093/annonc/mdq185

20. Swartz JE, Pothen AJ, Wegner I, Smid EJ, Swart KM, de Bree $R$ et al (2016) Feasibility of using head and neck CT imaging to assess skeletal muscle mass in head and neck cancer patients. Oral Oncol 62:28-33

21. Wendrich AW, Swartz JE, Bril SI, Wegner I, de Graeff A, Smid EJ et al (2017) Low skeletal muscle mass is a predictive factor for chemotherapy dose-limiting toxicity in patients with locally advanced head and neck cancer. Oral Oncol 71:26-33. https://doi. org/10.1016/j.oraloncology.2017.05.012

22. Bozkurt G, Elhassan HA, Mahmutoglu AS, Çelebi I, Mcleod RWJ, Soyta's P et al (2018) Neck muscle mass index as a predictor of post-laryngectomy wound complications. Ann Otol Rhinol Laryngol 127(11):841-847. https://doi.org/10.1177/0003489418 798660

23. Bril SI, Pezier TF, Tijink BM, Janssen LM, Braunius WW, de Bree R (2019) Preoperative low skeletal muscle mass as a risk factor for pharyngocutaneous fistula and decreased overall survival in patients undergoing total laryngectomy. Head and Neck. https:// doi.org/10.1002/hed.25638

24. Edge SB, Compton CC (2010) The American Joint Committee on Cancer: the 7th edition of the AJCC cancer staging manual and the future of TNM. Ann Surg Oncol 17(6):1471-1474. https://doi. org/10.1245/s10434-010-0985-4

25. Bol GH, Kotte A, Lagendijk JJW (2003) Volumetool. An image evaluation, registration, and delineation system for radiotherapy. Phys Med 19:80

26. Landis JR, Koch GG (1977) The measurement of observer agreement for categorical data. Biometrics 33(1):159-174
27. Shrout PE, Fleiss JL (1979) Intraclass correlations: uses in assessing rater reliability. Psychol Bull 86(2):420-428

28. Bland JM, Altman DG (1986) Statistical methods for assessing agreement between two methods of clinical measurement. Lancet 1(8476):307-310

29. Hebuterne X, Lemarie E, Michallet M, de Montreuil CB, Schneider SM, Goldwasser F (2014) Prevalence of malnutrition and current use of nutrition support in patients with cancer. J Parenter Enter Nutr 38(2):196-204. https://doi.org/10.1177/0148607113 502674

30. Beijer YJ, Koopman M, Terhaard CH, Braunius WW, van Es RJ, de Graeff A (2013) Outcome and toxicity of radiotherapy combi ned with chemotherapy or cetuximab for head and neck cancer: our experience in one hundred and twenty-five patients. Clin Otolaryngol 38(1):69-74. https://doi.org/10.1111/coa.12002

31. Achim V, Bash J, Mowery A, Guimaraes AR, Li R, Schindler $\mathrm{J}$ et al (2017) Prognostic indication of sarcopenia for wound complication after total laryngectomy. JAMA Otolaryngol Head Neck Surg 143(12):1159-1165. https://doi.org/10.1001/jamao to. 2017.0547

32. Sjoblom B, Benth JS, Gronberg BH, Baracos VE, Sawyer MB, Flotten O et al (2017) Drug dose per kilogram lean body mass predicts hematologic toxicity from carboplatin-doublet chemotherapy in advanced non-small-cell lung cancer. Clin Lung Cancer 18(2):e129-e136. https://doi.org/10.1016/j.cllc.2016.09.008

33. Huang J, Lai Y, Zhou X, Li S, Su J, Yang M et al (2017) Shortterm high-intensity rehabilitation in radically treated lung cancer: a three-armed randomized controlled trial. J Thorac Dis 9(7):1919-1929. https://doi.org/10.21037/jtd.2017.06.15

34. Dort JC, Farwell DG, Findlay M, Huber GF, Kerr P, Shea-Budgell MA et al (2017) Optimal perioperative care in major head and neck cancer surgery with free flap reconstruction: a consensus review and recommendations from the enhanced recovery after surgery society. JAMA Otolaryngol Head Neck Surg 143(3):292303. https://doi.org/10.1001/jamaoto.2016.2981

35. Lonbro S, Dalgas U, Primdahl H, Overgaard J, Overgaard K (2013) Feasibility and efficacy of progressive resistance training and dietary supplements in radiotherapy treated head and neck cancer patients-the DAHANCA 25A study. Acta Oncol 52(2):310-318. https://doi.org/10.3109/0284186X.2012.741325

36. Weed HG, Ferguson ML, Gaff RL, Hustead DS, Nelson JL, Voss AC (2011) Lean body mass gain in patients with head and neck squamous cell cancer treated perioperatively with a protein- and energy-dense nutritional supplement containing eicosapentaenoic acid. Head Neck 33(7):1027-1033. https://doi.org/10.1002/ hed. 21580

37. Argiris A, Karamouzis MV, Raben D, Ferris RL (2008) Head and neck cancer. Lancet 17 371(9625):1695-1709. https://doi. org/10.1016/S0140-6736(08)60728-X 